\title{
Maintenance of construction pit against hydrological influences
}

\author{
Yanis Olekhnovich $^{1 *}$, Kirill Sulatskiy ${ }^{1}$ and Kirill Kulakov ${ }^{2}$ \\ ${ }^{1}$ Peter the Great St. Petersburg Polytechnic University, Russian Federation \\ ${ }^{2}$ Moscow State University of Civil Engineering, Yaroslavskoe shosse, 26, Moscow, 129337, Russia
}

\begin{abstract}
During long-term operation, buildings and structures for various reasons receive various defects and damage in the form of precipitation and subsidence of the soil base, rolls of buildings and structures, deformations and displacements of the elements of the bearing base, which are recorded by geodetic methods. Traditionally, while examining buildings and structures, geodetic methods are based on optical instruments (theodolites, levels, total stations) and grades, which are installed on the elements of controlled buildings and structures are used. The indicated optical methods, on the one hand, give high accuracy in registering deformations of the foundations and structures in comparison with other known methods, and on the other hand, the most famous methods have a number of disadvantages. This article has collected and refined information about one of the most effective geodetic method for assessing the deformation of the structures
\end{abstract}

\section{Introduction}

The geodetic methods, which are used nowadays for examining the operational condition of the structure and and the rest of it's geodetic specifications, have much more advantages, than disadvantages, because these methods are the result of many years work. Modern geodetic methods include using different pieces of technologies, like theodolites, levels, and special techniques, which help to get the most accurate results with minimal labor and minimal amount of workers.

However, some wrong engineer's calculations can put all the process of making a project in bad position, because there are many factors, which can prevent the workers from getting right results, and some factors can be unknown for the workers until they understand that the results were wrong after collapse of the structure.

Fortunately for all people, who are responsible for the operational condition of the buildings and structures, new appliances recently started to appear in geodetic sphere, and one of them is Inclinometer.

Briefly, Inklinometer is an appliance, which us used to find the tilt angle of some part of the building. The information about using this type of geodetic appliances is gathered in this article [1-5].

\footnotetext{
${ }^{*}$ Corresponding author: openedu@spbstu.ru
} 


\section{Materials and Methods}

Modern optical methods, as was said in the introduction of this article, have several disadvantages.

Firstly, on critical construction sites, for example, on space rocket complexes (RKK), the use of these geodetic instruments requires the preliminary creation of a reference benchmark network, according to which the deformations of buildings and structures are controlled. In this case, the reference benchmark network, created from massive deep benchmarks, must meet the requirement of spatial stability (immobility), which requires periodic monitoring of the stability of the deep benchmarks [5].

Secondly, the ability to perform optical-geodetic measurements is significantly affected by climatic and weather conditions. Thirdly, the parameters of deformations and displacements of the bases and structures are only indirect signs of such a basic parameter of the technical condition of buildings and structures like the bearing capacity (NS) of their elements, which is evaluated according to two characteristics:

- By not exceeding the actual loads $R_{f}$ of the critical loads $R_{c r}$ :

$$
R_{f} \leq R_{c r}
$$

- By not exceeding the actual stresses $G_{f}$ the tensile strength of the structural material and soil $R$ :

$$
G_{f} \leq R
$$

The second of these criteria theoretically allows, within the elastic work of building structures and soil foundations (within the scope of Hooke's law) but relative deformations $\varepsilon$, which can be recorded by periodic optical-geodesic measurements, to determine the actual stresses $G_{f}$ in the structures and foundations of buildings (with the provided value of Young's modulus $E$, given for the materials and for the soil):

$$
G_{f}=E \cdot \varepsilon
$$

However, the identification of the parameter $E$ requires laboratory testing of samples of the material of structures and soil foundations of operated buildings and structures. In practice it is not always possible to conduct such tests during the examining the construction sites, so it is more acceptable to use another criterion for assessing the technical condition of buildings and structures, namely:

$$
\left(\xi_{f}\right) i \leq\left(\xi_{a d d}\right) i
$$

$\left(\xi_{f}\right) i$ and $\left(\xi_{\text {add }}\right) i$ are accordingly, the actual and permissible values of the $\mathrm{i}$-th parameters related to the deformation and displacements of structures, soil bases and buildings and structures in general [5].

In practice, the use of the value (3) is possible in those cases when, according to the parameters $\xi_{i}$ in the regulatory documents the values of $\xi_{\text {add }}$ are provided. It should be borne in mind that for deformations (for example, the strength of span structures), the permissible values ( $\xi$ add) $i$ are indicated from the condition that there is no loss of structural stability according to Euler, which is equivalent to condition (1) in an unclear form. However, according to the parameters $\xi \mathrm{i}$, for which acceptable values ( $\xi$ add) $\mathrm{i}$ have not been established, the use of criterion (3) is impossible.

Unfortunately, the optical - geodetic measurements are difficult to automatically register, and it does not meet the requirements of GOST R 22.1.12-2005 «Using automated monitoring systems (CM) for the technical condition of buildings and structures». In this regard, in recent years, inclinometers have been used more and more widely, allowing automatic recording of angular displacements of structures and rolls of buildings and 
structures. An inclinometer is a device designed to measure the angle of inclination of various objects relative to the gravitational field of the Earth. A rational arrangement of inclinometers allows you to determine for some types of structures not only their angular displacements, but also linear deformations [8]. You can clearly see the construction of the modern inclinometer in the Figure 1:
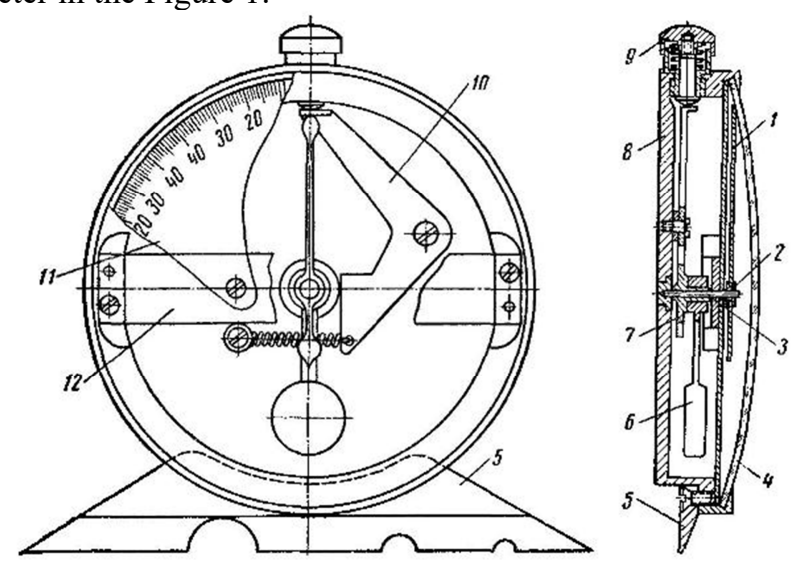

Fig. 1. The construction of the modern inclinometer.

A ruler 5 is screwed to the base of the body 8 of the protractor. A disk 7 with a plumb load 6 and an arrow 1 with a sleeve 2 are mounted on a freely rotating axis 3 . In the bore of the body 8 there is a plate 11 on which a graduated scale is laid out, divided into four parts, of which each has divisions from 0 to $45^{\circ}$, the division price is $1^{\circ}$. The plate 11 with the scale is screwed to the bridge 12 , mounted in the housing 8 . The scale is covered with glass. The spring-loaded brake lever 10 ensures the immobility of the axis 3 with the arrow 1 mounted on it at different positions of the protractor. Button 9 serves to release the axis 3 , load b and arrow 1.

The basis of the inclinometer is a pendulum. The scheme for measuring the angle of inclination using a pendulum type inclinometer is shown in the Figure 2:
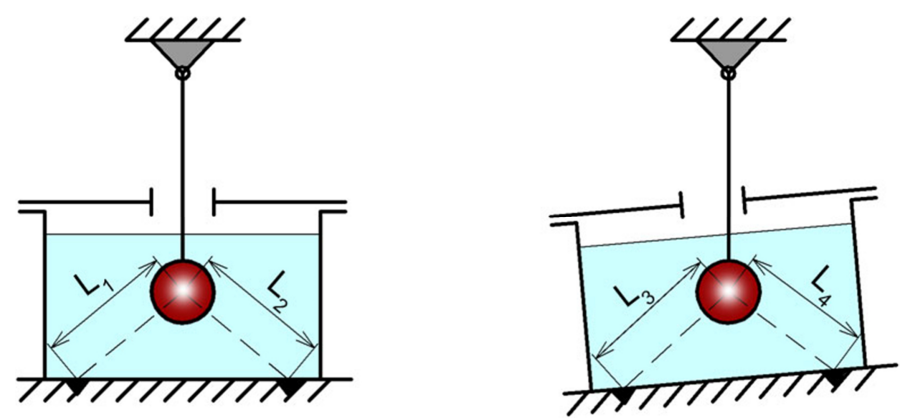

Fig. 2. The scheme of measuring the angle of inclination using the inclinometer.

$$
\text { L1 < L3; L2 > L4 [9]. }
$$

The pendulum is placed in a vessel with a conductive liquid, the electrodes are located on the base associated with the surface, the slope of which is measured. The pendulum, under the influence of gravity, will always be oriented vertically.

When measuring the angle of inclination, the distance between the pendulum and the electrodes will change, so the thickness of the liquid layer between the pendulum and the 
electrodes will also change, which will cause a change in the resistance, which is detected by electronic sensors, and transmitted to the secondary transducer.

Using the angle sensors, you can monitor the position of critical elements of structures and structures. A rational arrangement of inclinometers allows you to determine for some types of structures not only their angular displacements, but also linear deformations. So, if you install an inclinometer in the middle of the span on a span structure experiencing deflection $F$ (see inclinometer 3 in Figure 3), this inclinometer will always show a zero rotation in the middle of the span of the structure. But if inclinometers are installed near the reference sections of the span structure (see inclinometers 1 and 2 in Figure 3), these inclinometers will record the angles of inclination of the structure $\varphi$, from which it is easy to calculate its deflection $F$ in the middle of the span [1-3].

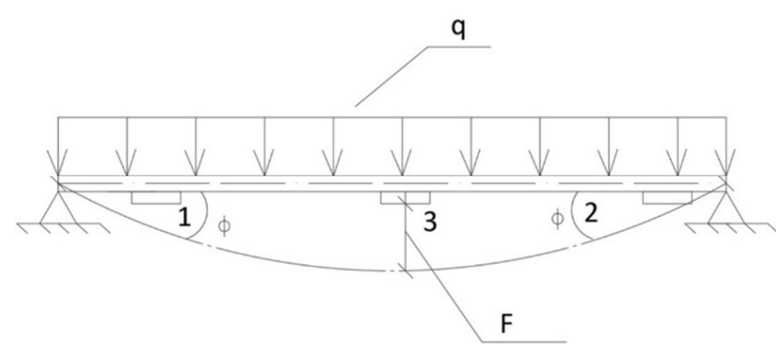

Fig. 3. The scheme of arrangement of the inclinometers on the span construction.

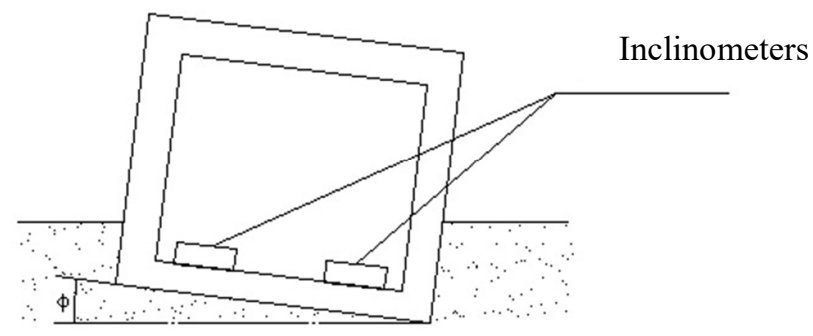

Fig. 4. The settlement of the inclinometers on the foundation for the registration of the sediment of the soil base.

Also, with the help of inclinometers, it is possible to fix the rolls of a rigid foundation slab of a building and structures, indicating the degree of uneven precipitation and subsidence of the soil base (see Figure 4), which in many cases is important information about the technical condition of buildings and structures, although it is impossible to register the sediment $S$ of the soil base with the help of inclinometers [9].

\section{Results}

In this article the information about the special geodetic device named inclinometer is shown, as the purpose of the article was to show the advantages of this device over the rest of the appliances, which are used nowadays to get the geodetic measurements.

Firstly, in the article there is an information about the principals of work of the inclinometer. All of the modern inclinometers have pendulum inside, so it makes the process of getting measurements more automated.

Secondly, the scheme of arrangement of the inclinometers on the span construction is made and shown in this article (Figure 3). According to this scheme, engineers, who would 
make a decision to use these devices in the project making process, can understand the rules and basics for getting proper measurements. Finally, according to the beginning of the article, there is an information, which can help to connect all the values, related to the geodetic measurements, between each other. It is necessary to follow the rules of exploring the safety of operational conditions of the building.

\section{Conclusion}

Nowadays geodetic workers should spend much time to get different measurements, which are necessary for beginning the project or it's continuation. The devices, which are used now, have been developed for many years, but sometimes it isn't enough to get proper results by spending minimal amount of strength. The workers of the geodetic sphere should get every small result independently, and it can take days, sometimes even months.

Inclinometers are the devices for more automated work, so it's very perspective sphere for making new inventions to make the getting more accurate results automatically. Modern inclinometers have pendulum and the scale, so, if the surveyor works with these devices, all of the results he determines by eye, and it's doesn't exempt the results from errors. In the perspective of development, the electric inclinometers would make the working process faster and the results, getting on this process, more accurate. Based on this, it can afford to minimize the labor of the surveyors. Currently, more and more new methods are being developed to assess the operational status of buildings and structures, and over time, measurements become automated, so the most widely used devices are automatically recording and measuring deformations and movements of elements of buildings and structures. The use of inclinometers allows engineers and surveyors to obtain more accurate measurement results, thus, due to a decrease in errors, more information will be obtained with the help of instruments for further research and making decisions.

Based on the analysis and research, the conclusion follows: inclinometers, being devices, translate geodetic measurements into an automated form and reduce the measurement error. Automated measurements reduce labor costs, so inclinometers serve as a step to further improve geodesy as a science and as a means of assessing the operation of buildings and structures.

\section{References}

1. Baylis A, Peters S, Van Leeuwen Z, E3S Web of Conferences Lyon (2016)

2. Li J, Liu T, Deng T, Tian M, MATEC Web of Conferences (Xiamen, China) (2016)

3. Lane S 2014 Hydrology and earth system sciences 318 pp 927-952

4. Ng L, Ong D, Wong W, Gannilegedera D 2015 Computer Methods and Recent Advances in Geomechan-ics pp 1205-1211 (Kyoto, Japan)

5. Zhang S, Jing F, Huang Y, Sun M Journal of Civil, Architectural and Environmental Engineering 38 pp 43-49 (2016)

6. Palla A, Colli M, Candela A, Aronica G, Lanza L, Journal of Flood Risk Management (Genoa, Italy) (2016)

7. $\mathrm{Hu} \mathrm{H}, \mathrm{Li} X$ China Civil Engineering Journal 48 pp 217-221 (2016)

8. Meng C, Xiong D, Zhao H, Zhang Y, Yu T 2014 Chinese Journal of Geotechnical Engineering 36265268

9. Krakovska A, Novakova J, Unucka J, Lapcfk V, Andras P, Klimsa L Carpathian Journal of Earth and Environmental Sciences 12 pp 283-292 (2017) 